\title{
Editorial
}

\section{Contrast Induced Nephropathy - CIN}

Contrast induced nephropathy (CIN) is a common cause of acute renal dysfunction particularly during cardiac intervention but also in many other radiological procedures. CIN is the acute deterioration of renal function after parenteral administration of radiocontrast media in the absence of other causes. CIN is generally defined as an increase in serum creatinine concentration of $>0.5 \mathrm{mg} / \mathrm{dL}(>44 \mu \mathrm{mol} / \mathrm{L})$ or $25 \%$ above baseline within 48 hours after contrast administration. The contrast medium was assumed to be the cause of the renal failure if it was administered in the 24 hours before renal failure and no other major kidney insult was identified.

Diabetes and pre existing renal impairment is a major risk factor for deterioration in renal function after angiography. Other factors variably associated with increased rates of acute renal failure after the administration of contrast medium include the elderly patient, volume depletion, heart failure, cirrhosis, hypertension, proteinuria, concomitant use of nonsteroidal antiinflammatory drugs and other drugs causing renal vasomotor changes. In the setting of acute myocardial infarction or percutaneous coronary intervention, hypotension or use of an aortic balloon pump has been associated with a higher rate of acute renal failure after exposure to a contrast medium. High doses of contrast medium also increase the likelihood of renal dysfunction. The tolerable dose of contrast medium depends on kidney function.

The pathogenesis of CIN is poorly understood. It is believed that intra renal vasoconstriction causes renal medullary hypoxia that culminates in cell detachment, apoptosis and necrosis. Radio contrast medium may directly damage tubular cells via the difference in osmolality or direct cytotoxicity and reperfusion injury may occur after initial tissue ischaemia via reactive oxygen species production.

Contrast induced nephropathy most commonly clinically manifests either as a non oliguric or an oliguric nephropathy. In the non oliguric variety there is a transient decline in renal function. Serum creatinine levels begin to rise within 24 hours of contrast administration peaking at 3-5 days and then returning to baseline in 10-15 days. In the oliguric variety, which is a more severe form, there is a drop in urine output to less than $400 \mathrm{ml}$ in 24 hours, serum creatinine levels peak in 5-10 days returning to normal in 14-21 days. In a small percentage the serum creatinine continues to rise, urine output drops, these individuals need then to be put on dialysis. Mortality from contrast induced nephropathy is also well documented.

A decline in kidney function after the administration of a contrast medium is associated with a prolonged hospital stay, adverse cardiac events, and high mortality both in the hospital and in the long term. However, the association between these outcomes and the decline in function may be explained at least in part by coexisting conditions, acuteness of illness, or other causes of acute kidney failure, such as atheroembolism.

Contrast induced nephropathy can be reduced by identify the person those who are at risk, whether the procedure is absolute necessary or not and use of low volume, iso osmolar non ionic contrast, adequate hydration of the patient with normal saline, avoidance of medication particularly NSAID, Aminoglycoside, ACE I, ARB etc. and close follow up of the patient. NAcetyl cystene pre and post procedure can also be used.

\section{Dr. Moniruzzaman}

Joint Editor 